\title{
To Develop a Virtual Model of Microscopic Quantum Gravity
}

\author{
U. V. Satya Seshavatharam ${ }^{1}$ and S. Lakshminarayana ${ }^{2}$ \\ ${ }^{1}$ Hon. Faculty, I-SERVE, S. No-42, Hitex Rd., Hitech city, Hyderabad-84, Telangana, India \\ ${ }^{2}$ Department of Nuclear Physics, Andhra University, Visakhapatnam-03, AP, India \\ Corresponding Emails: vedakavi@serveveda.org (or) sln@auvsp.edu.in
}

\begin{abstract}
We would like to suggest that, by considering three virtual gravitational constants assumed to be associated with gravitational, electromagnetic and strong interactions along with a strongly interacting virtual nuclear elementary charge, a workable model of final unification can be developed. In a verifiable approach, Newtonian gravitational constant and Fermi's weak coupling constant can be interrelated via nuclear and electromagnetic gravitational constants.
\end{abstract}

Keywords: final unification; fermi's weak coupling constant; newtonian gravitational constant; virtual electromagnetic gravitational constant; virtual nuclear gravitational constant; virtual nuclear elementary charge

\section{Introduction}

The most desirable cases of any unified description are:

a) To implement gravity in microscopic physics and to estimate the magnitude of Newtonian gravitational constant.

b) To develop a model of microscopic quantum gravity.

c) To simplify the complicated issues of known physics.

d) To predict new effects, arising from a combination of the fields inherent in the unified description.

In this context, in our earlier publications [1-12] and references therein, we suggested the role of two new gravitational constants associated with strong and electromagnetic interactions. Proceeding further, we also suggested the role of a new elementary charge associated with nuclear interactions and strong coupling constant $[13,14]$. In this paper, by considering the word 'virtual' and by refining and re-arranging the old semi empirical relations, we make a bold attempt to fit the Fermi's weak coupling constant and Newtonian gravitational constant $[15,16]$. Estimated magnitudes are $1.44021 \times 10^{-62} \mathrm{~J} . \mathrm{m}^{3}$ and $6.679856 \times 10^{-11} \mathrm{~m}^{3} \mathrm{~kg}^{-1} \mathrm{sec}^{-2}$ respectively.

It may be noted that, success of any unified model either depends on its physical/mathematical back ground or depends on its wide range of applications. It is clear from the below proposed applications ( 1 to 53) that we could satisfactorily fit the nuclear, electroweak and Newtonian gravitational constants through unified semi-empirical relations. This sincere attempt is to be reviewed and ascertained by the scientific community. We would like to appeal that, with respect to currently believed String theory and Quantum gravity models, proposed semi empirical relations and proposed assumptions, can be given some consideration in developing a 'workable model' of TOE. 


\section{Nomenclature and magnitudes}

1) $e_{e} \cong 1.602176565 \times 10^{-19} \mathrm{C}=$ Elementary charge associated with electromagnetic interaction

2) $e_{s} \cong 4.72058686 \times 10^{-19} \mathrm{C}=$ Estimated virtual nuclear elementary charge

3) $\varepsilon_{0} \cong 8.854187817 \times 10^{-19} \mathrm{~F} / \mathrm{m}=$ Permittivity of free space

4) $m_{n} \cong 1.674927471 \times 10^{-27} \mathrm{~kg}=$ Rest mass of neutron

5) $m_{p} \cong 1.672621777 \times 10^{-27} \mathrm{~kg}=$ Rest mass of proton

6) $m_{e} \cong 9.10938291 \times 10^{-31} \mathrm{~kg}=$ Rest mass of electron

7) $\hbar \cong 1.054571726 \times 10^{-34} \mathrm{~J} . \mathrm{sec} .=$ Reduced Planck's constant

8) $\mu_{p} \cong\left(e_{s} \hbar / 2 m_{p}\right) \cong 1.488142 \times 10^{-26} \mathrm{~J} . T e s l a^{-1}=$ Estimated magnetic dipole moment of proton

9) $\mu_{n} \cong \frac{\hbar}{2 m_{n}}\left(e_{s}-e_{e}\right) \cong 9.8171 \times 10^{-27} \mathrm{~J} . T e s l a^{-1}=$ Estimated magnetic dipole moment of neutron

10) $c \cong 2.99792458 \times 10^{8} \mathrm{~m} \cdot \mathrm{sec}^{-1}=$ Speed of light

11) $\alpha \cong 7.2973525698 \times 10^{-3}=$ Fine structure ratio

12) $\alpha_{s} \cong\left(e_{e} / e_{s}\right)^{2} \cong 0.1151937095=$ Estimated strong coupling constant

13) $G_{s} \cong 3.32956087 \times 10^{28} \mathrm{~m}^{3} \mathrm{~kg}^{-1} \mathrm{sec}^{-2}=$ Estimated virtual gravitational constant associated with nuclear or strong interaction

14) $G_{e} \cong 2.374335472 \times 10^{37} \mathrm{~m}^{3} \mathrm{~kg}^{-1} \mathrm{sec}^{-2}=$ Estimated virtual gravitational constant associated with electromagnetic interaction

15) $G_{N} \cong 6.679856 \times 10^{-11} \mathrm{~m}^{3} \mathrm{~kg}^{-1} \mathrm{sec}^{-2}=$ Estimated (virtual) gravitational constant associated with gravitational interaction

16) $G_{F} \cong 1.44021 \times 10^{-62} \mathrm{~J} . \mathrm{m}^{3}=$ Estimated Fermi's weak coupling constant

17) $R_{0} \cong\left(2 G_{s} m_{p} / c^{2}\right) \cong 1.239290976 \times 10^{-15} \mathrm{~m}=$ Estimated nuclear charge radius

18) $R_{p} \cong\left(\sqrt{2} G_{s} m_{p} / c^{2}\right) \cong 0.87631105 \times 10^{-15} \mathrm{~m}=$ Estimated root mean square radius of proton

\section{Three basic assumptions of final unification}

In our earlier publications, we proposed the following three assumptions. In this paper we review them with the word, 'virtual'.

Assumption-1: Magnitude of the virtual gravitational constant associated with the electromagnetic interaction is, $G_{e} \cong 2.374335472 \times 10^{37} \mathrm{~m}^{3} \mathrm{~kg}^{-1} \mathrm{sec}^{-2}$.

Assumption-2: Magnitude of the virtual gravitational constant associated with the strong interaction [17] is, $G_{s} \cong 3.32956087 \times 10^{28} \mathrm{~m}^{3} \mathrm{~kg}^{-1} \mathrm{sec}^{-2}$.

Assumption-3: There exists a strongly interacting virtual nuclear elementary charge, $e_{s} \cong\left(e_{e} / \sqrt{\alpha_{s}}\right) \cong 4.72058686 \times 10^{-19} \mathrm{C}$ where $e_{e}$ is the currently believed electromagnetic elementary charge and $\alpha_{s}$ is the currently believed strong coupling constant. Like quarks, the strong 
interaction elementary charge is experimentally undetectable and can also be called as 'invisible elementary nuclear charge'.

Note-1: $G_{e}$ can be estimated with, $G_{e} \cong\left(\frac{1}{2 \pi}\right)^{2}\left(\frac{m_{p}}{m_{e}}\right)^{2}\left(\frac{e_{e}^{2}}{4 \pi \varepsilon_{0} m_{e}^{2}}\right)$.

Note-2: $G_{s}$ can be estimated with, $G_{s} \cong \frac{\hbar^{2} c^{2}}{G_{e} m_{p} m_{e}^{3}} \cong\left(\frac{\hbar c}{G_{e} m_{e}^{2}}\right)\left(\frac{\hbar c}{m_{e} m_{p}}\right)$.

Note-3: $G_{N}$ can be estimated with, $G_{N} \cong\left(\frac{m_{e}}{m_{p}}\right)^{9}\left(\frac{G_{s}}{G_{e}}\right)\left(\frac{\hbar c}{m_{p}^{2}}\right)$.

\section{Important and interesting results}

Considering the following semi empirical results one can understand and validate the role of the proposed three assumptions.

\section{A) Ratio of rest mass of proton to electron:}

With reference to $G_{e}$ :

$$
\left(\frac{m_{p}}{m_{e}}\right) \cong 2 \pi \sqrt{\frac{4 \pi \varepsilon_{0} G_{e} m_{e}^{2}}{e_{e}^{2}}}
$$

With reference to $\left(G_{s}\right.$ and $\left.G_{e}\right)$ :

$$
\begin{aligned}
& \left(\frac{m_{p}}{m_{e}}\right) \cong\left(\frac{4 \pi \varepsilon_{0} G_{e} m_{e}^{2}}{e_{e}^{2}}\right) /\left(\frac{4 \pi \varepsilon_{0} G_{s} m_{p}^{2}}{e_{s}^{2}}\right) \cong\left(\frac{G_{s} m_{p}^{2}}{\hbar c}\right)\left(\frac{G_{e} m_{e}^{2}}{\hbar c}\right) \\
& \rightarrow \frac{m_{p}}{m_{e}} \cong\left(\frac{G_{e} e_{s}^{2}}{G_{s} e_{e}^{2}}\right)^{\frac{1}{3}} \cong\left(\left(\frac{G_{e}}{G_{s}}\right)\left(\frac{1}{\alpha_{s}}\right)\right)^{\frac{1}{3}} \cong\left(\left(\frac{G_{e}}{\hbar c}\right)\left(\frac{G_{s}}{\hbar c}\right) m_{p}^{4}\right)^{\frac{1}{3}} \\
& \Rightarrow m_{p} \cong\left(\left(\frac{\hbar c}{G_{e}}\right)\left(\frac{\hbar c}{G_{s}}\right) \frac{1}{m_{e}^{3}}\right) \text { and } m_{e} \cong\left(\left(\frac{\hbar c}{G_{e}}\right)\left(\frac{\hbar c}{G_{s}}\right) \frac{1}{m_{p}}\right)^{\frac{1}{3}}
\end{aligned}
$$

With reference to $\left(G_{e}\right.$ and $\left.G_{N}\right)$ :

$$
\begin{aligned}
& \left(\frac{m_{p}}{m_{e}}\right) \cong\left(\frac{G_{N}}{G_{e}}\right)^{\frac{1}{6}}\left(\frac{\hbar c}{G_{N} m_{e}^{2}}\right)^{\frac{1}{4}} \\
& \rightarrow m_{p} \cong\left(\frac{G_{N}}{G_{e}}\right)^{\frac{1}{6}} \sqrt{m_{e} \sqrt{\frac{\hbar c}{G_{N}}}} \text { and } m_{e} \cong\left(\frac{G_{e}}{G_{N}}\right)^{\frac{1}{3}} m_{p}^{2} \sqrt{\frac{G_{N}}{\hbar c}}
\end{aligned}
$$


With reference to $\left(G_{e}, G_{s}\right.$ and $\left.G_{N}\right)$ :

$$
\left(\frac{m_{p}}{m_{e}}\right) \cong\left(\frac{G_{s}}{G_{N}^{2 / 3} G_{e}^{1 / 3}}\right)^{\frac{1}{7}}
$$

B) Electron and proton rest masses with respect to Planck mass :

$$
\begin{aligned}
& m_{p} \cong\left(\frac{G_{N}^{3}}{G_{e}^{2} G_{s}}\right)^{\frac{1}{7}} \sqrt{\frac{\hbar c}{G_{N}}} \\
& m_{e} \cong\left(\frac{G_{N}^{11}}{G_{s}^{6} G_{e}^{5}}\right)^{\frac{1}{21}} \sqrt{\frac{\hbar c}{G_{N}}}
\end{aligned}
$$

C) Strong coupling constant [13]:

$$
\alpha_{s} \cong\left(\frac{e_{e}}{e_{s}}\right)^{2} \cong \frac{G_{e} m_{e}^{3}}{G_{s} m_{p}^{3}} \cong\left(\frac{m_{e}}{m_{p}}\right)\left(\frac{G_{e} m_{e}^{2}}{G_{s} m_{p}^{2}}\right) \cong\left(\frac{\hbar c}{G_{s} m_{p}^{2}}\right)^{2}
$$

This can be compared with a broad range of quoted values, 0.113 to 0.12 pertaining to various measurements.

D) Magnetic moment of proton [14]:

$$
\mu_{p} \cong \frac{e_{s} \hbar}{2 m_{p}} \cong 1.488142 \times 10^{-26}{\mathrm{~J} . \text { Tesla }^{-1}}^{-1}
$$

This can be compared with the recommended value of $1.4106067873 \times 10^{-26}$ 燡Tesla ${ }^{-1}$

E) Magnetic moment of neutron[14]:

$$
\mu_{n} \cong \frac{e_{s} \hbar}{2 m_{n}}-\frac{e_{e} \hbar}{2 m_{n}} \cong \frac{\hbar}{2 m_{n}}\left(e_{s}-e_{e}\right) \cong 9.8171 \times 10^{-27}{\mathrm{~J} . \mathrm{Tesla}^{-1}}^{-1}
$$

This can be compared with the recommended value of $9.66237 \times 10^{-27}$ 燡Tesla ${ }^{-1}$

F) Nuclear charge radius:

$$
R_{0} \cong \frac{2 G_{s} m_{p}}{c^{2}} \cong 1.239291 \times 10^{-15} \mathrm{~m}
$$

G) Root mean square radius of proton $[13,14,18,19]$ :

$$
R_{p} \cong \frac{\sqrt{2} G_{s} m_{p}}{c^{2}} \cong 0.8763111 \times 10^{-15} \mathrm{~m}
$$

This can be compared with the recommended value of $(0.8751 \pm 0.0061) \times 10^{-15}$ 爉 
H) Weak coupling angle [13]:

$$
\sin ^{2} \theta_{W} \cong \frac{4 \pi \varepsilon_{0} G_{s} m_{p} m_{e}}{e^{2}} \cong 0.219893
$$

This can be compared with electroweak model definition of, $\sin ^{2} \theta_{W} \cong\left\{1-\left(\frac{M_{W}^{2}}{M_{Z}^{2}}\right)\right\} \cong 1-\left(\frac{80385 \mathrm{MeV}}{91187 \mathrm{MeV}}\right)^{2} \cong 0.22289$.

I) Up and down quark mass ratio [13]:

$$
\left(\frac{m_{u}}{m_{d}}\right) \cong \sqrt{\frac{4 \pi \varepsilon_{0} G_{s} m_{p} m_{e}}{e^{2}}} \cong \sin \theta_{W} \cong 0.468353
$$

Note: With reference to PDG [13], $m_{u} \cong 2.2_{-0.4}^{+0.6} \mathrm{MeV}$ and $m_{d} \cong 4.7_{-0.5}^{+0.5} \mathrm{M} \mathrm{eV}$. Corresponding $\left(\frac{m_{u}}{m_{d}}\right) \cong 0.38$ to 0.58 . It is noticed that, $\sqrt{0.38 \times 0.58} \cong 0.4695$.

\section{J) Ground state potential energy of electron in Hydrogen atom:}

$$
\left(E_{\text {pot }}\right)_{\text {ground }} \cong\left(\frac{e_{e}^{2}}{4 \pi \varepsilon_{0} G_{s} m_{p} m_{e}}\right)\left(\frac{e_{e}^{2}}{4 \pi \varepsilon_{0}\left(G_{e} m_{e} / c^{2}\right)}\right) \cong-\left(\frac{e_{e}^{2}}{4 \pi \varepsilon_{0} G_{s} m_{p} m_{e}}\right)\left(\frac{e_{e}^{2} c^{2}}{4 \pi \varepsilon_{0} G_{e} m_{e}}\right)
$$

Note: Considering $\left(\frac{1}{2 n^{2}}\right)$ as a probability of finding electron in any orbit labeled with $n=1,2,3, .$. further research can be carried out. Bohr radius can be addressed with,

$$
a_{0} \cong\left(\frac{4 \pi \varepsilon_{0} G_{e} m_{e}^{2}}{e_{e}^{2}}\right)\left(\frac{G_{s} m_{p}}{c^{2}}\right) \cong\left(\frac{4 \pi \varepsilon_{0} G_{s} m_{p} m_{e}}{e_{e}^{2}}\right)\left(\frac{G_{e} m_{e}}{c^{2}}\right)
$$

K) Planck's constant:

$$
\begin{gathered}
h \cong \sqrt{\left(\frac{e_{s}^{2}}{4 \pi \varepsilon_{0} c}\right)\left(\frac{G_{e} m_{e}^{2}}{c}\right)} \cong\left(\frac{e_{s}}{e_{e}}\right) \sqrt{\left(\frac{e_{e}^{2}}{4 \pi \varepsilon_{0} c}\right)\left(\frac{G_{e} m_{e}^{2}}{c}\right)} \cong\left(\frac{G_{s} m_{p}^{2}}{\hbar c}\right) \sqrt{\left(\frac{e_{e}^{2}}{4 \pi \varepsilon_{0} c}\right)\left(\frac{G_{e} m_{e}^{2}}{c}\right)} \\
\rightarrow h c \cong \sqrt{\left(2 \pi G_{s} m_{p}^{2}\right) \sqrt{\left(\frac{e_{e}^{2}}{4 \pi \varepsilon_{0}}\right)\left(G_{e} m_{e}^{2}\right)}}
\end{gathered}
$$

Note: $\left(2 \pi G_{s} m_{p}^{2}\right)$ can be considered as characteristic constant connected with atomic nucleus and $\sqrt{\left(\frac{e_{e}^{2}}{4 \pi \varepsilon_{0}}\right)\left(G_{e} m_{e}^{2}\right)}$ can be can be considered as characteristic constant connected with revolving electron. 


\section{L) Magnetic flux quantum in super conductivity [20]:}

$$
\Phi_{0} \cong \frac{h}{2 e_{e}} \cong \frac{1}{2} \sqrt{\left(\frac{m_{p}}{m_{e}}\right)\left(\frac{\mu_{0}}{4 \pi}\right)\left(G_{s} m_{p}^{2}\right)}
$$

M) Atomic radii [21]:

Let, $m_{U} \cong 1.66054 \times 10^{-27} \mathrm{~kg}=$ Unified atomic mass unit.

$A=$ Atomic mass number.

1) Characteristic atomic radius, $R_{\text {atom }} \cong \frac{2 \sqrt{G_{s} G_{e}} m_{U}}{c^{2}} \cong 32.86 \mathrm{pm}$

2) Characteristic atomic radii, $R_{A} \approx A^{\frac{1}{3}}\left(\frac{2 \sqrt{G_{s} G_{e}} m_{U}}{c^{2}}\right) \approx A^{\frac{1}{3}} \times 32.86 \mathrm{pm}$

By considering the periodic arrangement of atoms, further research can be carried out.

\section{N) Photoelectric work functions [22]:}

Let, $W_{Z}=$ Photoelectric work function of $\mathrm{Z}$.

$$
W_{Z} \approx-f \times A^{\frac{1}{3}} \sqrt{\frac{G_{s} m_{p}^{2}}{G_{e} m_{e}^{2}}}\left(\frac{e_{e}^{2}}{8 \pi \varepsilon_{0} R_{A}}\right)
$$

where $R_{A}$ is the radius of atom, $A$ is the atomic mass number and factor $f \cong(3 \pm 0.5)$. See table-1. For the preparation of table, we consider, $f \cong 3.0$. From the table, it is possible to say that,

1) As atomic number increases, factor $f$ starts from $\sim 3.5$ and gradually reaches to $\sim 2.5$.

2) Actual atomic radii for light atoms seem to be less than the reference values and for heavy atoms, actual atomic radii seem to be higher than the reference values.

\section{O) Earth's magnetic dipole moment}

Planet's earth's magnetic dipole moment can be understood with:

$$
\begin{aligned}
\mu_{\text {earth }} & \cong\left(\frac{G_{N}}{G_{e}}\right)^{\frac{1}{4}} *\left(\frac{e_{e} G_{e} M_{\text {earth }}}{c}\right) \cong\left(\frac{e_{e}\left(G_{e}^{3} G_{N}\right)^{\frac{1}{4}} M_{\text {earth }}}{c}\right) \\
& \cong 9.8253 \times 10^{22}{\mathrm{~J} . \text { Tesla }^{-1}}^{-1}
\end{aligned}
$$

This can be compared with the estimated order of magnitude of Earth's magnetic dipole moment $8.2 \times 10^{22} \mathrm{~J} . T e s l a^{-1}$. Based on this relation, other solar planets, exo-planets and neutron star's "mass dependent" order of magnitude of 'magnetic dipole moments' can be estimated [23]. See table-3. It may be noted that, for 30 hot Jupiters, on an average, estimated value is roughly 0.21 times the reference values. See table -2 . 


\section{P) Neutron star mass and radius}

1) If $\left(M_{N S}, m_{n}\right)$ represent the masses of neutron star [24] and neutron, then,

$$
\frac{G_{N} M_{N S} m_{n}}{\hbar c} \approx \sqrt{\frac{G_{s}}{G_{N}}} \rightarrow M_{N S} \approx 3.175 M_{\odot}
$$

Note: By considering $\left(\frac{\hbar}{2}\right)$, mass of neutron star can be estimated to be $1.5875 M_{\odot}$. This is just greater than the famous Chandrasekhar mass limit of $1.4 M_{\odot}$.

2) If $R_{N S}$ represents the neutron star radius, then,

$$
\frac{R_{N S}}{\left(\sqrt{G_{s} \hbar / c^{3}}\right)} \approx \sqrt{\frac{G_{s}}{G_{N}}} \rightarrow R_{N S} \approx 8.06 \mathrm{~km}
$$

\section{To understand proton's melting point}

With reference to Hawking black hole temperature formula [25], melting point of proton [26] can be understood with:

$$
T_{\text {proton }} \cong \frac{\hbar c^{3}}{8 \pi k_{B} G_{s} m_{p}} \cong 0.15 \times 10^{12} \mathrm{~K}
$$

Based on this relation and with reference to up quark, other quark melting points can be expressed with the following kind of relation.

$$
T_{\text {quark }} \cong\left(\frac{m_{q}}{m_{u p}}\right) \frac{\hbar c^{3}}{8 \pi k_{B} G_{s} m_{u p}}
$$

where $\left(\frac{m_{q}}{m_{u p}}\right)$ represents the ratio of mass of any quark to mass of up quark. Based on this relation, for up quark of rest energy $2 \mathrm{MeV}$, its corresponding $T_{u p} \cong 69$ Tera $K$ and $8 \pi k_{B} T_{u p} \cong 236 \mathrm{MeV}$. This energy can be compared with currently believed QCD energy scale of $270 \mathrm{MeV}$.

\section{Fitting medium, heavy and super heavy nuclear charge radii}

For medium, heavy and super heavy atomic nuclei, nuclear charge radii [27,28,29] can be fitted with the following simple relation.

$$
\begin{aligned}
R_{(Z, A)} & \cong\left\{Z^{1 / 3}+(\sqrt{Z(A-Z)})^{1 / 3}\right\}\left(\frac{G_{s} m_{p}}{c^{2}}\right) \\
& \cong\left\{Z^{1 / 3}+[Z(A-Z)]^{1 / 6}\right\}\left(\frac{G_{s} m_{p}}{c^{2}}\right)
\end{aligned}
$$


where $Z=(2$ to 100$)$ and $\left(G_{s} m_{p} / c^{2}\right) \cong 0.62 \mathrm{fm}$

It may be noted that, this relation is free from arbitrary numbers and can be compared with the following relation available in recent literature. See table-3.

$$
R_{(Z, N)} \cong\left\{1-0.349\left(\frac{N-Z}{N}\right)\right\} N^{\frac{1}{3}} 1.262 \mathrm{fm}
$$

\section{Fitting and Understanding Fermi's weak coupling constant}

Quantitatively the famous Fermi's weak coupling constant $[13,14]$ can be fitted with the following relation.

When distance between two protons is close to $R_{0} \cong \frac{2 G_{s} m_{p}}{c^{2}} \cong 1.234 \mathrm{fm}$, gravitational force of attraction between them can be expressed as,

$$
\frac{G_{s} m_{p} m_{p}}{R_{0}^{2}} \cong \frac{G_{s} m_{p}^{2}}{R_{0}^{2}} \cong\left(\frac{c^{4}}{4 G_{s}}\right)
$$

Based on this idea, $F_{W}$ can be expressed in the following ways.

$$
\begin{aligned}
G_{F} & \cong\left(\frac{e_{e}}{e_{s}}\right)\left(G_{s} m_{p}^{2}\right)\left(G_{s} m_{e}^{2}\right)\left(\frac{c^{4}}{4 G_{s}}\right)^{-1} \cong\left(\frac{e_{e}}{e_{s}}\right)\left(G_{s} m_{e}^{2} R_{0}^{2}\right) \\
& \cong \sqrt{\alpha_{s}}\left(G_{s} m_{e}^{2} R_{0}^{2}\right) \cong\left(\frac{m_{e}}{m_{p}}\right)^{2}\left(\hbar c R_{0}^{2}\right) \cong \hbar c\left(\frac{2 G_{s} m_{e}}{c^{2}}\right)^{2} \\
& \cong\left[\left(G_{e} m_{p}^{2}\right)^{2}\left(G_{N} m_{p}^{2}\right)\right]^{\frac{1}{3}}\left(\frac{2 G_{s} m_{p}}{c^{2}}\right)^{2} \\
& \cong 1.44021 \times 10^{-62} \mathrm{~J} . \mathrm{m}^{3}
\end{aligned}
$$

It may be noted that, this relation is free from all numerical factors and accuracy mainly depends on $\left[\sqrt{\alpha_{s}}\right.$ or $\left.\left(\frac{e_{e}}{e_{s}}\right)\right]$ and $R_{0}$. It is for further study. Based on these relations,

$$
G_{N} \cong \frac{G_{F}^{3} c^{12}}{64 G_{e}^{2} G_{s}^{6} m_{p}^{12}}
$$

If, recommended $G_{F} \cong 1.435850984 \times 10^{-62} \mathrm{~J}^{3}$, obtained $G_{N} \cong 6.619386 \times 10^{-11} \mathrm{~m}^{3} \mathrm{~kg}^{-1} \mathrm{sec}^{-2}$

\section{Fitting \& Understanding nuclear stability and binding energy}

A) Proton-Neutron stability: 
Let,

$$
s \cong\left(\frac{G_{s} m_{p} m_{e}}{\hbar c}\right) \cong\left(\frac{\hbar c}{G_{e} m_{e}^{2}}\right) \cong\left[\left(\frac{e_{s}}{m_{p}}\right) /\left(\frac{e_{e}}{m_{e}}\right)\right] \cong 1.604637101 \times 10^{-3}
$$

Clearly speaking, $s$ is the ratio of specific charge of proton associated with $e_{s}$ to specific charge of electron associated with $e_{e}$. Using this ratio, proton-neutron stability relation can be fitted directly in the following way [30].

$$
A_{s} \cong 2 Z+k(2 Z)^{2} \cong 2 Z+(4 s) Z^{2} \cong 2 Z+0.00641855 Z^{2}
$$

where $A_{s}$ is the estimated stable mass number of $Z$.

It is very interesting to note that, close to beta stability line, considering $(4 s) \cong k \cong 0.00641855$, nuclear binding energy [31-36] can be estimated very easily.

\section{B) Nuclear binding energy:}

Nuclear binding energy potential can be expressed with the following relation.

$$
\begin{aligned}
& B_{0} \cong \sqrt{\left(\frac{e_{s}^{2}}{4 \pi \varepsilon_{0} \hbar c}\right)\left(\frac{e^{2}}{4 \pi \varepsilon_{0} \hbar c}\right)}\left(\frac{G_{s} m_{p}^{2}}{R_{0}}\right) \cong\left(\frac{e_{e} e_{s}}{4 \pi \varepsilon_{0} \hbar c}\right)\left(\frac{G_{s} m_{p}^{2}}{R_{0}}\right) \\
& \cong\left(\frac{G_{s} m_{p}^{2}}{\hbar c}\right)\left(\frac{e_{e} e_{s}}{4 \pi \varepsilon_{0} R_{0}}\right) \cong\left(\frac{e_{s}^{2}}{4 \pi \varepsilon_{0} R_{0}}\right) \cong\left(\frac{e_{s}^{2} c^{2}}{8 \pi \varepsilon_{0} G_{s} m_{p}}\right) \cong 10.0867 \mathrm{MeV}
\end{aligned}
$$

Based on the new integrated model proposed by N. Ghahramany et al [31,32] and with reference to relation (31), it is possible to show that, $Z \cong(40$ to 83$)$, close to the beta stability line,

$$
(B)_{A_{s}} \cong\left[A_{s}-\left(\frac{N_{s}^{2}-Z^{2}}{3 Z}\right)\right] \times 9.5 \mathrm{MeV} \cong\left[A_{s}-\left(\frac{k Z A_{s}}{3}\right)\right] \times 9.5 \mathrm{MeV}
$$

where, $\left[\frac{N_{s}^{2}-Z^{2}}{Z}\right] \cong k Z A_{s}$. Based on this strange and simple relation and with reference to our recent publication [37] and first four terms of the semi empirical mass formula (SEMF), close to the beta stability line, for $(Z=2$ to 100$)$, it is possible to show that,

$$
(B)_{A_{s}} \cong\left[A_{s}-A_{s}^{1 / 3}-\frac{k A_{s} \sqrt{N_{s} Z}}{3.40}-1\right] \times\left(B_{0} \cong 10.09 \mathrm{MeV}\right)
$$

This can be compared with first four terms of the semi empirical mass formula where : $a_{v} \cong 15.77 \mathrm{MeV}, a_{s} \cong 18.34 \mathrm{MeV}, a_{a} \cong 23.21 \mathrm{MeV}$ and $a_{c} \cong 0.71 \mathrm{MeV}$. See table-4. For relation (34), see figure 1 (dashed red curve) for the estimated binding energy per nucleon close to beta stability 
line of $Z=2$ to 100 compared with first four terms of the semi empirical mass formula (Green curve).

For $Z=50$ and $A=100$ to 136 , estimated binding energy range is (857 to 1140 ) $\mathrm{MeV}$ and can be compared with reference binding energy [38] range of (806 to 1105) $\mathrm{MeV}$. It is for further study. With reference to SEMF, close to the beta stability line, it is also possible to show that,

$$
\frac{\left(A_{s}-2 Z\right)^{2}}{A_{s}} \cong\left(k^{2} A_{s} N_{s} \sqrt{Z}\right)
$$

Let, $\left\{\begin{array}{l}a_{v} \cong a_{s} \cong a_{a} \approx 14.8 \mathrm{MeV} \approx(3 / 2) \times 10.0 \mathrm{MeV} \\ \text { and } a_{c} \cong 0.71 \mathrm{MeV}\end{array}\right\}$.

If so,

$$
(B)_{A_{s}} \approx\left[A_{s}-A_{s}^{2 / 3}-0.0473\left[\frac{Z(Z-1)}{A_{s}^{1 / 3}}\right]-\left(k^{2} A_{s} N_{s} \sqrt{Z}\right)\right] \times 14.8 \mathrm{MeV}
$$

In comparison with SEMF, by replacing $A_{s}$ with $A$ and $N_{s}$ with $(A-Z)$ in relation (36) and by considering a multiplication factor of the kind $\left(A_{S} / A\right)^{1-(Z / A)}$ associated with each term, binding energy of $A$ can be estimated approximately.

\section{Neutron life time and Avogadro number}

Neutron life time [39] can be fitted in the following way,

$$
\begin{aligned}
& \left(m_{n}-m_{p}\right) c^{2} \times t_{n} \cong \sqrt{\frac{G_{e}}{G_{N}}}\left(\frac{G_{s} m_{n}^{2}}{c}\right) \\
& \rightarrow t_{n} \cong \sqrt{\frac{G_{e}}{G_{N}}}\left(\frac{G_{s} m_{n}^{2}}{\left(m_{n}-m_{p}\right) c^{3}}\right) \cong 896.8 \mathrm{sec}
\end{aligned}
$$

where, $\sqrt{\frac{G_{e}}{G_{N}}} \cong 5.964517556 \times 10^{23}$ is very close to the Avogadro number, $N_{A}[40,41]$.

By considering the unified atomic mass unit $m_{U} \cong 1.66054 \times 10^{-27} \mathrm{~kg}$,

$$
\rightarrow t_{n} \cong \sqrt{\frac{G_{e}}{G_{N}}}\left(\frac{G_{s} m_{U}^{2}}{\left(m_{n}-m_{p}\right) c^{3}}\right) \cong 878.6 \mathrm{sec}
$$

By considering the unified atomic mass unit $m_{U} \cong 1.66054 \times 10^{-27} \mathrm{~kg}$ as well as neutron proton rest masses,

$$
\rightarrow t_{n} \cong \sqrt{\frac{G_{e}}{G_{N}}}\left(\frac{G_{s} m_{U} \sqrt{m_{p} m_{n}}}{\left(m_{n}-m_{p}\right) c^{3}}\right) \cong 888.5 \mathrm{sec}
$$

In this way, results of bottle experiments and beam experiments both can be fitted [13]. 


\section{Strange results connected with Planck scale Schwarzschild radius}

A) Conceptual thought: Schwarzschild radius of Planck mass plays a vital role in electroweak and strong interactions.

$$
\begin{aligned}
& \text { Planck mass }=M_{p l} \cong \sqrt{\frac{\hbar c}{G_{N}}} \\
& R_{p l} \cong \text { Schwarzschild radius of Planck mass } \\
& \cong \frac{2 G_{N} M_{p l}}{c^{2}} \cong 2 \sqrt{\frac{G_{N} \hbar}{c^{3}}}
\end{aligned}
$$

B) Strange result connected with $\left(G_{F}\right.$ and $\left.G_{N}\right)$

It is noticed that

$$
\left(\frac{m_{p}}{m_{e}}\right) \cong\left(\frac{G_{F}}{\hbar c R_{p l}^{2}}\right)^{\frac{1}{10}} \cong\left(\frac{G_{F} c^{2}}{4 G_{N} \hbar^{2}}\right)^{\frac{1}{10}}
$$

Based on this relation,

$$
\begin{gathered}
G_{F} \cong\left(\frac{m_{p}}{m_{e}}\right)^{10}\left(\frac{4 G_{N} \hbar^{2}}{c^{2}}\right) \\
G_{N} \cong\left(\frac{m_{e}}{m_{p}}\right)^{10}\left(\frac{G_{F} c^{2}}{4 \hbar^{2}}\right)
\end{gathered}
$$

If, recommended $G_{F} \cong 1.435850984 \times 10^{-62} \mathrm{~J} \cdot \mathrm{m}^{3}$, obtained $G_{N} \cong 6.65963739 \times 10^{-11} \mathrm{~m}^{3} \mathrm{~kg}^{-1} \mathrm{sec}^{-2}$

If, estimated $G_{F} \cong 1.44021 \times 10^{-62} \mathrm{~J} . \mathrm{m}^{3}$, obtained $G_{N} \cong 6.679856 \times 10^{-11} \mathrm{~m}^{3} \mathrm{~kg}^{-1} \mathrm{sec}^{-2}$

If, $G_{N} \cong\left(\frac{m_{e}}{m_{p}}\right)^{9}\left(\frac{G_{s}}{G_{e}}\right)\left(\frac{\hbar c}{m_{p}^{2}}\right)$, from relation (43), it is also possible to show that,

$$
\begin{aligned}
& G_{F} \cong\left(\frac{\hbar c}{G_{e} m_{p} m_{e}}\right) \hbar c\left(2 \sqrt{\frac{G_{s} \hbar}{c^{3}}}\right)^{2} \cong\left(\frac{G_{s}}{G_{e}}\right)\left(\frac{4 \hbar^{3}}{m_{p} m_{e} c}\right) \cong\left(\frac{G_{s}}{G_{e}}\right) 4 \hbar c\left(\frac{\hbar}{m_{p} c}\right)\left(\frac{\hbar}{m_{e} c}\right) \\
& \text { where, } 2 \sqrt{\frac{G_{s} \hbar}{c^{3}}} \cong 0.722 \mathrm{fm}=\text { Schwarzschild radius of } \sqrt{\frac{\hbar c}{G_{s}} \cong \frac{2 G_{s}}{c^{2}} \sqrt{\frac{\hbar c}{G_{s}}}} .
\end{aligned}
$$

\section{C) Strange result connected with Proton-electron mass ratio}

From above relations, 


$$
\begin{gathered}
\frac{R_{0}}{R_{p l}} \cong\left(\frac{m_{p}}{m_{e}}\right)^{6} \text { (Or) } \frac{\pi R_{0}^{2}}{\pi R_{p l}^{2}} \cong\left(\frac{m_{p}}{m_{e}}\right)^{12} \\
R_{0} \cong \frac{2 G_{s} m_{p}}{c^{2}} \cong\left(\frac{m_{p}}{m_{e}}\right)^{6} R_{s p l} \cong\left(\frac{m_{p}}{m_{e}}\right)^{6}\left(2 \sqrt{\frac{G_{N} \hbar}{c^{3}}}\right) \\
\rightarrow G_{N} \cong\left(\frac{G_{s} m_{p}^{2}}{\hbar c}\right)\left(\frac{m_{e}}{m_{p}}\right)^{12} G_{s} \cong\left(\frac{1}{\sqrt{\alpha_{s}}}\right)\left(\frac{m_{e}}{m_{p}}\right)^{12} G_{s} \\
\text { If }\left(\frac{1}{\sqrt{\alpha_{s}}}\right) \cong\left(\frac{G_{s} m_{p}^{2}}{\hbar c}\right), \text { obtained, } G_{N} \cong 6.6798563 \times 10^{-11} \mathrm{~m}^{3} \mathrm{~kg}^{-1} \mathrm{sec}^{-2}
\end{gathered}
$$

\section{1.'System of units' independent Avogadro number and Molar mass unit}

If, atoms as a whole believed to exhibit electromagnetic interaction, then molar mass constant and Avogadro number, both can be understood with the following simple relation.

$$
G_{e}\left(m_{\text {atom }}\right)^{2} \cong G_{N}\left(M_{\text {mole }}\right)^{2}
$$

Where $m_{\text {atom }}$ is the unified atomic mass unit and $M_{\text {mole }}$ is the molar mass unit or gram mole.

Thus it is very clear to say that, directly and indirectly 'gravity' plays a key role in understanding the molar mass unit.

$$
\begin{aligned}
& \frac{M_{\text {mole }}}{m_{\text {atom }}} \cong \sqrt{\frac{G_{e}}{G_{N}}} \rightarrow M_{\text {mole }} \cong \sqrt{\frac{G_{e}}{G_{N}}} \times m_{\text {atom }} \\
& \text { where } \sqrt{\frac{G_{e}}{G_{N}}} \cong 5.96 \times 10^{23} \cong \text { Avogadro number, } N_{A} \\
& \text { and }\left(0.00099>M_{\text {mole }}<0.001\right) \mathrm{kg}
\end{aligned}
$$

Based on these relations, "independent of system of units" and "independent of ad-hoc selection of exactly one gram or one kilogram", it may be possible to explore the correct physical meaning of the famous 'Molar mass unit' and 'Avogadro number' in a unified approach. It may be noted that, Avogadro number and 'gram mole' are having many applications in solid state physics, gas dynamics/thermodynamics and basic chemistry/electrochemistry. By considering the following relation, it is possible to couple the Avogadro number with the observed fundamental interactions.

$$
\sqrt{\frac{M_{p l} * m_{e}}{m_{p}^{2}}} \cong\left(\frac{G_{e}}{G_{N}}\right)^{\frac{1}{6}} \cong N_{A}^{1 / 3}
$$




\section{Relation in between $\left(G_{s}, G_{e}, G_{N}\right.$ and $\left.\alpha\right)$ :}

It may be noted that, fitting the gravitational constant with elementary physical constants is a very challenging issue. G. Rosi et al say [15]: "There is no definitive relationship between $G_{N}$ and the other fundamental constants, and there is no theoretical prediction for its value, against which to test experimental results. Improving the precision with which we know $G_{N}$ has not only a pure metrological interest, but is also important because of the key role that $G_{N}$ has in theories of gravitation, cosmology, particle physics and astrophysics and in geophysical models".

In this context, we would like to stress the fact, with currently available standard theoretical models, it may not be possible to fit and verify the Newtonian gravitational constant with elementary physical constants. With the following semi empirical relations and with further research, in a verifiable approach, it is certainly possible to explore the back ground physics of the role of the Newtonian gravitational constant in microscopic physics.

In a semi empirical approach it is also noticed that,

$$
\begin{aligned}
& \ln \left(\frac{G_{s}^{4}}{G_{e}^{2} G_{N}^{2}}\right) \approx \ln \left[\left(\frac{G_{s}^{2}}{G_{e}^{2}}\right)\left(\frac{G_{s}^{2}}{G_{N}^{2}}\right)\right] \cong 137.44057 \approx \frac{1}{\alpha} \\
& \rightarrow G_{N} \cong 8.170389 \times 10^{-11} \mathrm{~m}^{3} \mathrm{~kg}^{-1} \mathrm{sec}^{-2} .
\end{aligned}
$$

With reference to the recommended and experimental values of the Newtonian gravitational constant, with trial-error and by introducing an ad hoc factor of $(3 / 2)$, it is noticed that,

$$
\begin{gathered}
\left(\frac{G_{s}^{4}}{G_{e}^{2} G_{N}^{2}}\right) \cong \frac{3}{2} \exp \left(\frac{1}{\alpha}\right) \\
G_{N} \cong \sqrt{\left(\frac{2}{3}\right)\left[\exp \left(\frac{1}{\alpha}\right)\right]^{-1} \times \frac{G_{s}^{4}}{G_{e}^{2}}} \cong 6.6710946 \times 10^{-11} \mathrm{~m}^{3} \mathrm{~kg}^{-1} \mathrm{sec}^{-2} .
\end{gathered}
$$

The ad-hoc fitting factor (3/2) needs a detailed explanation and is for further study.

\section{Discussion}

It is true that, unless stringent requirements are met, in general, speculative alternatives to currently accepted theories cannot be accepted or published. Scientific papers having content that lie outside the mainstream of current research must justify by including a clear, detailed discussion of the motivation for the new speculation, with reasons for introducing any new concepts. If the new formulation results are in contradiction with the accepted theory, then there must both be a discussion of which experiments could be done to verify that the conventional theory needs improvement, and also an analysis showing the consistency of the new theory with the existing experiments. In this context, we appeal that, 
1) Subject of final unification is having a long unsuccessful history. Clearly speaking, so far, no model succeeded in implementing the Newtonian gravitational constant or Planck scale in nuclear and electroweak interactions.

2) Even though, the basic idea of String theory $[42,43]$ is very simple, very interesting and highly intuitive, there are no concrete new predictions on low energy scales and high energy scale predictions are beyond the reach of current technology.

3) It may be noted that, since 1992, J. E Brandenburg is working on 'GEM unification theory' [44] and proposed an interesting and unified relation, $\frac{e^{2}}{4 \pi \varepsilon_{0} G_{N} m_{p} m_{e}} \cong\left(\frac{1}{\alpha}\right)\left\{\exp \sqrt{\frac{m_{p}}{m_{e}}}\right\}^{2}$. Compared to J. E Brandenburg and other available models of current unification theories, in this paper, with reference to Planck scale, we presented a variety of multipurpose arithmetic relations pertaining to nuclear, electroweak and astrophysical applications.

4) As the current unification paradigm is failing in developing a 'practical unification procedure', the point that we wish to emphasize here is that, by considering three new assumptions, we presented a number of applications connecting micro-macro physical systems and finally developed arithmetic relations for understanding the role of the Newtonian gravitational constant in microscopic physics. We appeal the mainstream physicists to see the possibility of considering the proposed relations for further investigation with respect to strong, electroweak and gravitational interactions collectively.

5) Following this kind of computational approach, it is certainly possible to reproduce another set of arithmetic relations by using which, in near future, it may be possible to find a set of absolute relations having sound physical reasoning and strong mathematical back up.

\section{Conclusion}

As it is inevitable to unite gravity and other three atomic interactions, if one is willing to explore the possibility of incorporating the proposed assumptions either in String theory models or in Quantum gravity models, certainly, background physics assumed to be connected with proposed semi empirical relations can be understood and in near future, a 'workable' or 'practical' model of "everything" can be developed. Based on relations (28) and (29), Fermi's weak coupling constant and the three gravitational constants can be fitted in a unified approach and finally, Newtonian gravitational constant can be estimated accurately with microscopic physical constants.

\section{Acknowledgements}

Author Seshavatharam U.V.S is indebted to professors brahmasri Dr. M. Nagaphani Sarma, Chairman, shri K.V. Krishna Murthy, former chairman, Institute of Scientific Research in Vedas (I-SERVE), Hyderabad, India and Shri K.V.R.S. Murthy, former scientist IICT (CSIR), Govt. of India, Director, Research and Development, I-SERVE, for their valuable guidance and great support in developing this subject. 
Table-1: To fit and estimate the photo electric work functions

\begin{tabular}{|c|c|c|c|c|c|}
\hline $\begin{array}{l}\text { Proton } \\
\text { number }\end{array}$ & $\begin{array}{l}\text { Mass number } \\
\text { close to beta } \\
\text { stability line }\end{array}$ & Symbol & $\begin{array}{l}\text { Atomic } \\
\text { Radius } \\
(\mathrm{pm})\end{array}$ & $\begin{array}{c}\text { Estimated } \\
\text { work } \\
\text { function }(\mathrm{eV})\end{array}$ & $\begin{array}{c}\text { Experimental } \\
\text { work } \\
\text { function }(\mathrm{eV})\end{array}$ \\
\hline 3 & 7 & $\mathrm{Li}$ & $1.28 \mathrm{E}-10$ & 2.22 & 2.9 \\
\hline 4 & 9 & $\mathrm{Be}$ & $9.60 \mathrm{E}-11$ & 3.22 & 4.98 \\
\hline 5 & 10 & B & $8.40 \mathrm{E}-11$ & 3.81 & 4.45 \\
\hline 6 & 12 & $\mathrm{C}$ & $7.60 \mathrm{E}-11$ & 4.47 & 5 \\
\hline 11 & 23 & $\mathrm{Na}$ & $1.66 \mathrm{E}-10$ & 2.54 & 2.36 \\
\hline 12 & 25 & $\mathrm{Mg}$ & $1.41 \mathrm{E}-10$ & 3.08 & 3.66 \\
\hline 13 & 27 & $\mathrm{Al}$ & $1.21 \mathrm{E}-10$ & 3.68 & 4.28 \\
\hline 14 & 29 & $\mathrm{Si}$ & $1.11 \mathrm{E}-10$ & 4.11 & 4.85 \\
\hline 19 & 40 & $\mathrm{~K}$ & $2.03 \mathrm{E}-10$ & 2.50 & 2.29 \\
\hline 20 & 43 & $\mathrm{Ca}$ & $1.76 \mathrm{E}-10$ & 2.96 & 2.87 \\
\hline 21 & 45 & $\mathrm{Sc}$ & $1.70 \mathrm{E}-10$ & 3.11 & 3.5 \\
\hline 22 & 47 & $\mathrm{Ti}$ & $1.60 \mathrm{E}-10$ & 3.35 & 4.33 \\
\hline 23 & 49 & $\mathrm{~V}$ & $1.53 \mathrm{E}-10$ & 3.55 & 4.3 \\
\hline 24 & 52 & $\mathrm{Cr}$ & $1.39 \mathrm{E}-10$ & 3.99 & 4.5 \\
\hline 25 & 54 & $\mathrm{Mn}$ & $1.39 \mathrm{E}-10$ & 4.04 & 4.1 \\
\hline 26 & 56 & $\mathrm{Fe}$ & $1.32 \mathrm{E}-10$ & 4.30 & 4.5 \\
\hline 27 & 59 & $\mathrm{Co}$ & $1.26 \mathrm{E}-10$ & 4.59 & 5 \\
\hline 28 & 61 & $\mathrm{Ni}$ & $1.24 \mathrm{E}-10$ & 4.71 & 5.15 \\
\hline 29 & 63 & $\mathrm{Cu}$ & $1.32 \mathrm{E}-10$ & 4.48 & 4.51 \\
\hline 31 & 68 & $\mathrm{Ga}$ & $1.22 \mathrm{E}-10$ & 4.97 & 4.32 \\
\hline 33 & 73 & As & $1.19 \mathrm{E}-10$ & 5.22 & 3.75 \\
\hline 34 & 75 & $\mathrm{Se}$ & $1.20 \mathrm{E}-10$ & 5.22 & 5.9 \\
\hline 37 & 83 & $\mathrm{Rb}$ & $2.20 \mathrm{E}-10$ & 2.94 & 2.26 \\
\hline 38 & 85 & $\mathrm{Sr}$ & $1.95 \mathrm{E}-10$ & 3.35 & 2.59 \\
\hline 39 & 88 & $\mathrm{Y}$ & $1.90 \mathrm{E}-10$ & 3.48 & 3.1 \\
\hline 40 & 90 & $\mathrm{Zr}$ & $1.75 \mathrm{E}-10$ & 3.80 & 4.05 \\
\hline 41 & 93 & $\mathrm{Nb}$ & $1.64 \mathrm{E}-10$ & 4.10 & 4.3 \\
\hline 42 & 95 & Mo & $1.54 \mathrm{E}-10$ & 4.40 & 4.6 \\
\hline 44 & 100 & $\mathrm{Ru}$ & $1.46 \mathrm{E}-10$ & 4.72 & 4.71 \\
\hline 45 & 103 & $\mathrm{Rh}$ & $1.42 \mathrm{E}-10$ & 4.90 & 4.98 \\
\hline 46 & 106 & $\mathrm{Pd}$ & $1.39 \mathrm{E}-10$ & 5.06 & 5.12 \\
\hline 47 & 108 & $\mathrm{Ag}$ & $1.45 \mathrm{E}-10$ & 4.88 & 4.26 \\
\hline 48 & 111 & $\mathrm{Cd}$ & $1.44 \mathrm{E}-10$ & 4.96 & 4.08 \\
\hline 49 & 113 & In & $1.42 \mathrm{E}-10$ & 5.06 & 4.09 \\
\hline 50 & 116 & $\mathrm{Sn}$ & $1.39 \mathrm{E}-10$ & 5.21 & 4.42 \\
\hline 51 & 119 & $\mathrm{Sb}$ & $1.39 \mathrm{E}-10$ & 5.26 & 4.55 \\
\hline 52 & 121 & $\mathrm{Te}$ & $1.39 \mathrm{E}-10$ & 5.28 & 4.95 \\
\hline 55 & 129 & $\mathrm{Cs}$ & $2.44 \mathrm{E}-10$ & 3.08 & 1.95 \\
\hline 56 & 132 & $\mathrm{Ba}$ & $2.15 \mathrm{E}-10$ & 3.52 & 2.7 \\
\hline 57 & 135 & $\mathrm{La}$ & $2.07 \mathrm{E}-10$ & 3.68 & 3.5 \\
\hline 58 & 138 & $\mathrm{Ce}$ & $2.04 \mathrm{E}-10$ & 3.76 & 2.9 \\
\hline 60 & 143 & $\mathrm{Nd}$ & $2.01 \mathrm{E}-10$ & 3.86 & 3.2 \\
\hline 62 & 149 & $\mathrm{Sm}$ & $1.98 \mathrm{E}-10$ & 3.98 & 2.7 \\
\hline
\end{tabular}




\begin{tabular}{|c|c|c|c|c|c|}
\hline 63 & 151 & $\mathrm{Eu}$ & $1.98 \mathrm{E}-10$ & 3.99 & 2.5 \\
\hline 64 & 154 & $\mathrm{Gd}$ & $1.96 \mathrm{E}-10$ & 4.06 & 3.17 \\
\hline 65 & 157 & $\mathrm{~Tb}$ & $1.94 \mathrm{E}-10$ & 4.13 & 3.15 \\
\hline 66 & 160 & Dy & $1.92 \mathrm{E}-10$ & 4.20 & 3.25 \\
\hline 67 & 163 & Ho & $1.92 \mathrm{E}-10$ & 4.23 & 3.22 \\
\hline 68 & 166 & $\mathrm{Er}$ & $1.89 \mathrm{E}-10$ & 4.32 & 3.25 \\
\hline 69 & 169 & $\mathrm{Tm}$ & $1.89 \mathrm{E}-10$ & 4.34 & 3.1 \\
\hline 70 & 171 & $\mathrm{Yb}$ & $1.87 \mathrm{E}-10$ & 4.41 & 3 \\
\hline 71 & 174 & $\mathrm{Lu}$ & $1.87 \mathrm{E}-10$ & 4.43 & 3.3 \\
\hline 72 & 177 & Hf & $1.75 \mathrm{E}-10$ & 4.76 & 3.9 \\
\hline 73 & 180 & $\mathrm{Ta}$ & $1.70 \mathrm{E}-10$ & 4.93 & 4.25 \\
\hline 74 & 183 & W & $1.62 \mathrm{E}-10$ & 5.20 & 4.55 \\
\hline 75 & 186 & $\mathrm{Re}$ & $1.51 \mathrm{E}-10$ & 5.61 & 4.72 \\
\hline 76 & 189 & Os & $1.44 \mathrm{E}-10$ & 5.92 & 5.93 \\
\hline 77 & 192 & Ir & $1.41 \mathrm{E}-10$ & 6.08 & 5.27 \\
\hline 78 & 195 & $\mathrm{Pt}$ & $1.36 \mathrm{E}-10$ & 6.33 & 5.65 \\
\hline 79 & 198 & $\mathrm{Au}$ & $1.36 \mathrm{E}-10$ & 6.36 & 5.1 \\
\hline 80 & 201 & $\mathrm{Hg}$ & $1.32 \mathrm{E}-10$ & 6.59 & 4.49 \\
\hline 81 & 204 & $\mathrm{Tl}$ & $1.45 \mathrm{E}-10$ & 6.03 & 3.84 \\
\hline 82 & 207 & $\mathrm{~Pb}$ & $1.46 \mathrm{E}-10$ & 6.02 & 4.25 \\
\hline 83 & 210 & $\mathrm{Bi}$ & $1.48 \mathrm{E}-10$ & 5.96 & 4.34 \\
\hline 84 & 213 & Po & $1.40 \mathrm{E}-10$ & 6.34 & 5 \\
\hline 90 & 232 & $\mathrm{Th}$ & $2.06 \mathrm{E}-10$ & 4.43 & 3.4 \\
\hline 91 & 235 & $\mathrm{~Pa}$ & $2.00 \mathrm{E}-10$ & 4.58 & 3.7 \\
\hline 92 & 238 & $\mathrm{U}$ & $1.96 \mathrm{E}-10$ & 4.70 & 3.63 \\
\hline 93 & 242 & $\mathrm{~Np}$ & $1.90 \mathrm{E}-10$ & 4.87 & 3.9 \\
\hline 94 & 245 & $\mathrm{Pu}$ & $1.87 \mathrm{E}-10$ & 4.97 & 3.6 \\
\hline 95 & 248 & $\mathrm{Am}$ & $1.87 \mathrm{E}-10$ & 4.99 & 3.7 \\
\hline 96 & 251 & $\mathrm{Cm}$ & $1.69 \mathrm{E}-10$ & 5.54 & 3.9 \\
\hline
\end{tabular}

Table-2: To fit the mass dependent magnetic dipole moments of hot Jupiters

\begin{tabular}{|c|c|c|c|c|}
\hline Hot Jupiter & $\begin{array}{c}\text { Mass } \\
(\mathrm{kg})\end{array}$ & $\begin{array}{c}\text { Magnetic dipole } \\
\text { moment data taken } \\
\text { from ref. [23] } \\
\text { (J/tesla) }\end{array}$ & $\begin{array}{c}\text { Estimated } \\
\text { magnetic dipole } \\
\text { moment from } \\
\text { relation (20) }\end{array}$ & $\begin{array}{c}\text { Ratio of } \\
\text { estimated } \\
\text { value to } \\
\text { ref. value }\end{array}$ \\
\hline HD 160691 d & $7.98 \mathrm{E}+25$ & $1.89 \mathrm{E}+24$ & $1.31 \mathrm{E}+24$ & 0.694 \\
\hline 55 CnC e & $8.55 \mathrm{E}+25$ & $7.91 \mathrm{E}+24$ & $1.40 \mathrm{E}+24$ & 0.178 \\
\hline GJ 436 b & $1.27 \mathrm{E}+26$ & $1.31 \mathrm{E}+25$ & $2.09 \mathrm{E}+24$ & 0.159 \\
\hline HD 49674 b & $2.28 \mathrm{E}+26$ & $1.25 \mathrm{E}+25$ & $3.75 \mathrm{E}+24$ & 0.300 \\
\hline HD 76700 b & $3.74 \mathrm{E}+26$ & $2.76 \mathrm{E}+25$ & $6.14 \mathrm{E}+24$ & 0.223 \\
\hline HD 88133 b & $4.18 \mathrm{E}+26$ & $3.69 \mathrm{E}+25$ & $6.87 \mathrm{E}+24$ & 0.186 \\
\hline HD 168746 b & $4.37 \mathrm{E}+26$ & $1.93 \mathrm{E}+25$ & $7.18 \mathrm{E}+24$ & 0.372 \\
\hline HD 46375 b & $4.73 \mathrm{E}+26$ & $4.84 \mathrm{E}+25$ & $7.77 \mathrm{E}+24$ & 0.161 \\
\hline HD 63454 b & $7.22 \mathrm{E}+26$ & $8.35 \mathrm{E}+25$ & $1.19 \mathrm{E}+25$ & 0.142 \\
\hline HD 83443 b & $7.79 \mathrm{E}+26$ & $8.52 \mathrm{E}+25$ & $1.28 \mathrm{E}+25$ & 0.150 \\
\hline HD 75289 b & $7.98 \mathrm{E}+26$ & $7.31 \mathrm{E}+25$ & $1.31 \mathrm{E}+25$ & 0.179 \\
\hline 51 Peg b & $8.89 \mathrm{E}+26$ & $6.71 \mathrm{E}+25$ & $1.46 \mathrm{E}+25$ & 0.218 \\
\hline
\end{tabular}




\begin{tabular}{|c|c|c|c|c|} 
BD -10 3166 b & $9.12 \mathrm{E}+26$ & $8.54 \mathrm{E}+25$ & $1.50 \mathrm{E}+25$ & 0.175 \\
\hline HD 2638 b & $9.12 \mathrm{E}+26$ & $8.66 \mathrm{E}+25$ & $1.50 \mathrm{E}+25$ & 0.173 \\
\hline HD 187123 b & $9.88 \mathrm{E}+26$ & $1.06 \mathrm{E}+26$ & $1.62 \mathrm{E}+25$ & 0.153 \\
\hline OGLE-TR-111 b & $1.01 \mathrm{E}+27$ & $8.15 \mathrm{E}+25$ & $1.66 \mathrm{E}+25$ & 0.204 \\
\hline OGLE-TR-10 b & $1.08 \mathrm{E}+27$ & $1.18 \mathrm{E}+26$ & $1.77 \mathrm{E}+25$ & 0.150 \\
\hline TrES-1 & $1.16 \mathrm{E}+27$ & $1.30 \mathrm{E}+26$ & $1.91 \mathrm{E}+25$ & 0.147 \\
\hline ups-And b & $1.31 \mathrm{E}+27$ & $9.35 \mathrm{E}+25$ & $2.15 \mathrm{E}+25$ & 0.230 \\
\hline HD 209458 b & $1.31 \mathrm{E}+27$ & $1.26 \mathrm{E}+26$ & $2.15 \mathrm{E}+25$ & 0.171 \\
\hline HD 330075 b & $1.44 \mathrm{E}+27$ & $1.48 \mathrm{E}+26$ & $2.37 \mathrm{E}+25$ & 0.160 \\
\hline HD 179949 b & $1.86 \mathrm{E}+27$ & $2.15 \mathrm{E}+26$ & $3.06 \mathrm{E}+25$ & 0.142 \\
\hline HD 130322 b & $2.05 \mathrm{E}+27$ & $6.04 \mathrm{E}+25$ & $3.37 \mathrm{E}+25$ & 0.558 \\
\hline OGLE-TR-132 b & $2.26 \mathrm{E}+27$ & $5.19 \mathrm{E}+26$ & $3.71 \mathrm{E}+25$ & 0.072 \\
\hline HD 217107 b & $2.43 \mathrm{E}+27$ & $1.15 \mathrm{E}+26$ & $3.99 \mathrm{E}+25$ & 0.347 \\
\hline OGLE-TR-113 b & $2.56 \mathrm{E}+27$ & $7.17 \mathrm{E}+26$ & $4.21 \mathrm{E}+25$ & 0.059 \\
\hline OGLE-TR-56 b & $2.75 \mathrm{E}+27$ & $9.34 \mathrm{E}+26$ & $4.52 \mathrm{E}+25$ & 0.048 \\
\hline HD 73256 b & $3.55 \mathrm{E}+27$ & $5.44 \mathrm{E}+26$ & $5.83 \mathrm{E}+25$ & 0.107 \\
\hline HD 68988 b & $3.61 \mathrm{E}+27$ & $2.04 \mathrm{E}+26$ & $5.93 \mathrm{E}+25$ & 0.291 \\
\hline Tau-Boo & $7.84 \mathrm{E}+27$ & $9.77 \mathrm{E}+26$ & $1.29 \mathrm{E}+26$ & 0.132 \\
\hline HD 162020 b & $2.61 \mathrm{E}+28$ & $1.32 \mathrm{E}+27$ & $4.29 \mathrm{E}+26$ & 0.325 \\
\hline
\end{tabular}

Table-3: To fit nuclear charge radii

\begin{tabular}{|c|c|c|c|c|}
\hline $\begin{array}{l}\text { Proton } \\
\text { number }\end{array}$ & $\begin{array}{c}\text { Mass } \\
\text { number }\end{array}$ & $\begin{array}{l}\text { Neutron } \\
\text { number }\end{array}$ & $\begin{array}{l}\text { Estimated } \\
\text { charge radii } \\
\text { from relation } \\
(24) \\
\text { fm }\end{array}$ & $\begin{array}{l}\text { Charge radii } \\
\text { from relation } \\
\text { (25) } \\
\text { fm }\end{array}$ \\
\hline 2 & 4 & 2 & 1.5623 & 1.5900 \\
\hline 3 & 6 & 3 & 1.7884 & 1.8201 \\
\hline 4 & 8 & 4 & 1.9684 & 2.0033 \\
\hline 5 & 10 & 5 & 2.1204 & 2.1580 \\
\hline 6 & 12 & 6 & 2.2532 & 2.2932 \\
\hline 7 & 14 & 7 & 2.3720 & 2.4141 \\
\hline 8 & 16 & 8 & 2.4800 & 2.5240 \\
\hline 9 & 19 & 10 & 2.6022 & 2.6240 \\
\hline 10 & 21 & 11 & 2.6929 & 2.7176 \\
\hline 11 & 23 & 12 & 2.7779 & 2.8052 \\
\hline 12 & 25 & 13 & 2.8580 & 2.8877 \\
\hline 13 & 27 & 14 & 2.9338 & 2.9658 \\
\hline 14 & 29 & 15 & 3.0059 & 3.0399 \\
\hline 15 & 31 & 16 & 3.0746 & 3.1107 \\
\hline 16 & 34 & 18 & 3.1556 & 3.1791 \\
\hline 17 & 36 & 19 & 3.2182 & 3.2438 \\
\hline 18 & 38 & 20 & 3.2785 & 3.3060 \\
\hline 19 & 40 & 21 & 3.3366 & 3.3660 \\
\hline 20 & 43 & 23 & 3.4055 & 3.4256 \\
\hline 21 & 45 & 24 & 3.4596 & 3.4814 \\
\hline 22 & 47 & 25 & 3.5119 & 3.5356 \\
\hline 23 & 49 & 26 & 3.5628 & 3.5881 \\
\hline 24 & 52 & 28 & 3.6233 & 3.6411 \\
\hline 25 & 54 & 29 & 3.6712 & 3.6906 \\
\hline 26 & 56 & 30 & 3.7178 & 3.7389 \\
\hline 27 & 59 & 32 & 3.7734 & 3.7881 \\
\hline 28 & 61 & 33 & 3.8176 & 3.8339 \\
\hline 29 & 63 & 34 & 3.8608 & 3.8786 \\
\hline
\end{tabular}




\begin{tabular}{|c|c|c|c|c|}
\hline 30 & 66 & 36 & 3.9124 & 3.9246 \\
\hline 31 & 68 & 37 & 3.9536 & 3.9673 \\
\hline 32 & 71 & 39 & 4.0027 & 4.0116 \\
\hline 33 & 73 & 40 & 4.0421 & 4.0524 \\
\hline 34 & 75 & 41 & 4.0808 & 4.0924 \\
\hline 35 & 78 & 43 & 4.1269 & 4.1342 \\
\hline 36 & 80 & 44 & 4.1640 & 4.1726 \\
\hline 37 & 83 & 46 & 4.2083 & 4.2130 \\
\hline 38 & 85 & 47 & 4.2440 & 4.2500 \\
\hline 39 & 88 & 49 & 4.2866 & 4.2891 \\
\hline 40 & 90 & 50 & 4.3211 & 4.3247 \\
\hline 41 & 93 & 52 & 4.3622 & 4.3627 \\
\hline 42 & 95 & 53 & 4.3955 & 4.3971 \\
\hline 43 & 98 & 55 & 4.4352 & 4.4339 \\
\hline 44 & 100 & 56 & 4.4674 & 4.4672 \\
\hline 45 & 103 & 58 & 4.5058 & 4.5029 \\
\hline 46 & 106 & 60 & 4.5436 & 4.5382 \\
\hline 47 & 108 & 61 & 4.5743 & 4.5699 \\
\hline 48 & 111 & 63 & 4.6109 & 4.6043 \\
\hline 49 & 113 & 64 & 4.6408 & 4.6351 \\
\hline 50 & 116 & 66 & 4.6764 & 4.6686 \\
\hline 51 & 119 & 68 & 4.7114 & 4.7016 \\
\hline 52 & 121 & 69 & 4.7400 & 4.7311 \\
\hline 53 & 124 & 71 & 4.7741 & 4.7633 \\
\hline 54 & 127 & 73 & 4.8077 & 4.7952 \\
\hline 55 & 129 & 74 & 4.8352 & 4.8235 \\
\hline 56 & 132 & 76 & 4.8679 & 4.8547 \\
\hline 57 & 135 & 78 & 4.9002 & 4.8854 \\
\hline 58 & 138 & 80 & 4.9320 & 4.9159 \\
\hline 59 & 140 & 81 & 4.9582 & 4.9428 \\
\hline 60 & 143 & 83 & 4.9893 & 4.9725 \\
\hline 61 & 146 & 85 & 5.0200 & 5.0020 \\
\hline 62 & 149 & 87 & 5.0503 & 5.0312 \\
\hline 63 & 151 & 88 & 5.0753 & 5.0568 \\
\hline 64 & 154 & 90 & 5.1050 & 5.0853 \\
\hline 65 & 157 & 92 & 5.1343 & 5.1136 \\
\hline 66 & 160 & 94 & 5.1633 & 5.1416 \\
\hline 67 & 163 & 96 & 5.1919 & 5.1693 \\
\hline 68 & 166 & 98 & 5.2202 & 5.1968 \\
\hline 69 & 168 & 99 & 5.2436 & 5.2207 \\
\hline 70 & 171 & 101 & 5.2714 & 5.2476 \\
\hline 71 & 174 & 103 & 5.2988 & 5.2743 \\
\hline 72 & 177 & 105 & 5.3260 & 5.3007 \\
\hline 73 & 180 & 107 & 5.3529 & 5.3269 \\
\hline 74 & 183 & 109 & 5.3795 & 5.3528 \\
\hline 75 & 186 & 111 & 5.4058 & 5.3785 \\
\hline 76 & 189 & 113 & 5.4319 & 5.4040 \\
\hline 77 & 192 & 115 & 5.4577 & 5.4293 \\
\hline 78 & 195 & 117 & 5.4833 & 5.4544 \\
\hline 79 & 198 & 119 & 5.5086 & 5.4792 \\
\hline 80 & 201 & 121 & 5.5337 & 5.5038 \\
\hline 81 & 204 & 123 & 5.5586 & 5.5282 \\
\hline 82 & 207 & 125 & 5.5832 & 5.5524 \\
\hline
\end{tabular}




\begin{tabular}{|l|l|l|l|l|}
\hline 83 & 210 & 127 & 5.6077 & 5.5765 \\
\hline 84 & 213 & 129 & 5.6319 & 5.6003 \\
\hline 85 & 216 & 131 & 5.6558 & 5.6239 \\
\hline 86 & 219 & 133 & 5.6796 & 5.6474 \\
\hline 87 & 222 & 135 & 5.7032 & 5.6706 \\
\hline 88 & 226 & 138 & 5.7302 & 5.6969 \\
\hline 89 & 229 & 140 & 5.7534 & 5.7198 \\
\hline 90 & 232 & 142 & 5.7763 & 5.7425 \\
\hline 91 & 235 & 144 & 5.7991 & 5.7651 \\
\hline 92 & 238 & 146 & 5.8217 & 5.7875 \\
\hline 93 & 241 & 148 & 5.8442 & 5.8097 \\
\hline 94 & 245 & 151 & 5.8698 & 5.8349 \\
\hline 95 & 248 & 153 & 5.8919 & 5.8568 \\
\hline 96 & 251 & 155 & 5.9138 & 5.8785 \\
\hline 97 & 254 & 157 & 5.9355 & 5.9001 \\
\hline 98 & 257 & 159 & 5.9570 & 5.9215 \\
\hline 99 & 261 & 162 & 5.9817 & 5.9459 \\
\hline 100 & 264 & 164 & 6.0029 & 5.9670 \\
\hline
\end{tabular}

Table 4: To estimate nuclear binding energy close to beta stability line

\begin{tabular}{|c|c|c|c|c|c|c|}
\hline $\begin{array}{l}\text { Proton } \\
\text { number }\end{array}$ & $\begin{array}{c}\text { Mass } \\
\text { number }\end{array}$ & $\begin{array}{l}\text { Neutron } \\
\text { number }\end{array}$ & $\begin{array}{c}\text { Estimated } \\
\text { binding } \\
\text { energy } \\
\text { Relation (34) } \\
\text { (MeV) }\end{array}$ & $\begin{array}{c}\text { Estimated } \\
\text { binding } \\
\text { energy per } \\
\text { nucleon } \\
(\mathrm{MeV})\end{array}$ & $\begin{array}{l}(1 \text { to } 4) \\
\text { terms of } \\
\text { SEMF } \\
\text { binding } \\
\text { energy } \\
(\mathrm{MeV})\end{array}$ & $\begin{array}{c}\text { (1 to } 4 \text { ) terms } \\
\text { of SEMF } \\
\text { binding } \\
\text { energy per } \\
\text { nucleon } \\
(\mathrm{MeV})\end{array}$ \\
\hline 2 & 4 & 2 & 14.1 & 3.53 & 16.0 & 3.99 \\
\hline 3 & 6 & 3 & 31.8 & 5.30 & 31.7 & 5.29 \\
\hline 4 & 8 & 4 & 49.8 & 6.23 & 48.5 & 6.07 \\
\hline 5 & 10 & 5 & 68.1 & 6.81 & 66.0 & 6.60 \\
\hline 6 & 12 & 6 & 86.5 & 7.21 & 83.8 & 6.98 \\
\hline 7 & 14 & 7 & 105.0 & 7.50 & 101.9 & 7.28 \\
\hline 8 & 16 & 8 & 123.5 & 7.72 & 120.1 & 7.51 \\
\hline 9 & 19 & 10 & 151.3 & 7.96 & 148.7 & 7.82 \\
\hline 10 & 21 & 11 & 169.8 & 8.08 & 167.3 & 7.97 \\
\hline 11 & 23 & 12 & 188.3 & 8.18 & 185.9 & 8.08 \\
\hline 12 & 25 & 13 & 206.7 & 8.27 & 204.5 & 8.18 \\
\hline 13 & 27 & 14 & 225.1 & 8.34 & 223.0 & 8.26 \\
\hline 14 & 29 & 15 & 243.5 & 8.40 & 241.4 & 8.32 \\
\hline 15 & 31 & 16 & 261.9 & 8.45 & 259.7 & 8.38 \\
\hline 16 & 34 & 18 & 289.3 & 8.51 & 288.4 & 8.48 \\
\hline 17 & 36 & 19 & 307.5 & 8.54 & 306.7 & 8.52 \\
\hline 18 & 38 & 20 & 325.7 & 8.57 & 324.9 & 8.55 \\
\hline 19 & 40 & 21 & 343.8 & 8.59 & 343.0 & 8.57 \\
\hline 20 & 43 & 23 & 370.9 & 8.62 & 371.1 & 8.63 \\
\hline 21 & 45 & 24 & 388.8 & 8.64 & 389.1 & 8.65 \\
\hline 22 & 47 & 25 & 406.7 & 8.65 & 407.0 & 8.66 \\
\hline 23 & 49 & 26 & 424.6 & 8.66 & 424.7 & 8.67 \\
\hline 24 & 52 & 28 & 451.2 & 8.68 & 452.4 & 8.70 \\
\hline 25 & 54 & 29 & 468.9 & 8.68 & 470.0 & 8.70 \\
\hline 26 & 56 & 30 & 486.5 & 8.69 & 487.4 & 8.70 \\
\hline 27 & 59 & 32 & 512.9 & 8.69 & 514.6 & 8.72 \\
\hline
\end{tabular}




\begin{tabular}{|c|c|c|c|c|c|c|}
\hline 28 & 61 & 33 & 530.4 & 8.69 & 531.9 & 8.72 \\
\hline 29 & 63 & 34 & 547.7 & 8.69 & 549.0 & 8.71 \\
\hline 30 & 66 & 36 & 573.7 & 8.69 & 575.8 & 8.72 \\
\hline 31 & 68 & 37 & 591.0 & 8.69 & 592.8 & 8.72 \\
\hline 32 & 71 & 39 & 616.7 & 8.69 & 619.1 & 8.72 \\
\hline 33 & 73 & 40 & 633.8 & 8.68 & 635.9 & 8.71 \\
\hline 34 & 75 & 41 & 650.8 & 8.68 & 652.5 & 8.70 \\
\hline 35 & 78 & 43 & 676.2 & 8.67 & 678.5 & 8.70 \\
\hline 36 & 80 & 44 & 693.0 & 8.66 & 694.9 & 8.69 \\
\hline 37 & 83 & 46 & 718.1 & 8.65 & 720.5 & 8.68 \\
\hline 38 & 85 & 47 & 734.8 & 8.64 & 736.7 & 8.67 \\
\hline 39 & 88 & 49 & 759.7 & 8.63 & 762.0 & 8.66 \\
\hline 40 & 90 & 50 & 776.1 & 8.62 & 778.0 & 8.64 \\
\hline 41 & 93 & 52 & 800.8 & 8.61 & 802.9 & 8.63 \\
\hline 42 & 95 & 53 & 817.0 & 8.60 & 818.8 & 8.62 \\
\hline 43 & 98 & 55 & 841.4 & 8.59 & 843.4 & 8.61 \\
\hline 44 & 100 & 56 & 857.5 & 8.58 & 859.0 & 8.59 \\
\hline 45 & 103 & 58 & 881.6 & 8.56 & 883.3 & 8.58 \\
\hline 46 & 106 & 60 & 905.6 & 8.54 & 907.4 & 8.56 \\
\hline 47 & 108 & 61 & 921.4 & 8.53 & 922.8 & 8.54 \\
\hline 48 & 111 & 63 & 945.1 & 8.51 & 946.5 & 8.53 \\
\hline 49 & 113 & 64 & 960.7 & 8.50 & 961.7 & 8.51 \\
\hline 50 & 116 & 66 & 984.2 & 8.48 & 985.2 & 8.49 \\
\hline 51 & 119 & 68 & 1007.5 & 8.47 & 1008.5 & 8.47 \\
\hline 52 & 121 & 69 & 1022.8 & 8.45 & 1023.4 & 8.46 \\
\hline 53 & 124 & 71 & 1045.8 & 8.43 & 1046.4 & 8.44 \\
\hline 54 & 127 & 73 & 1068.7 & 8.41 & 1069.2 & 8.42 \\
\hline 55 & 129 & 74 & 1083.7 & 8.40 & 1083.8 & 8.40 \\
\hline 56 & 132 & 76 & 1106.3 & 8.38 & 1106.4 & 8.38 \\
\hline 57 & 135 & 78 & 1128.8 & 8.36 & 1128.7 & 8.36 \\
\hline 58 & 138 & 80 & 1151.1 & 8.34 & 1150.9 & 8.34 \\
\hline 59 & 140 & 81 & 1165.7 & 8.33 & 1165.2 & 8.32 \\
\hline 60 & 143 & 83 & 1187.8 & 8.31 & 1187.1 & 8.30 \\
\hline 61 & 146 & 85 & 1209.6 & 8.29 & 1208.8 & 8.28 \\
\hline 62 & 149 & 87 & 1231.3 & 8.26 & 1230.4 & 8.26 \\
\hline 63 & 151 & 88 & 1245.6 & 8.25 & 1244.4 & 8.24 \\
\hline 64 & 154 & 90 & 1267.0 & 8.23 & 1265.7 & 8.22 \\
\hline 65 & 157 & 92 & 1288.3 & 8.21 & 1286.9 & 8.20 \\
\hline 66 & 160 & 94 & 1309.4 & 8.18 & 1307.9 & 8.17 \\
\hline 67 & 163 & 96 & 1330.4 & 8.16 & 1328.7 & 8.15 \\
\hline 68 & 166 & 98 & 1351.2 & 8.14 & 1349.5 & 8.13 \\
\hline 69 & 169 & 100 & 1371.9 & 8.12 & 1370.0 & 8.11 \\
\hline 70 & 171 & 101 & 1385.4 & 8.10 & 1383.4 & 8.09 \\
\hline 71 & 174 & 103 & 1405.7 & 8.08 & 1403.7 & 8.07 \\
\hline 72 & 177 & 105 & 1426.0 & 8.06 & 1423.9 & 8.04 \\
\hline 73 & 180 & 107 & 1446.0 & 8.03 & 1443.9 & 8.02 \\
\hline 74 & 183 & 109 & 1466.0 & 8.01 & 1463.8 & 8.00 \\
\hline 75 & 186 & 111 & 1485.7 & 7.99 & 1483.6 & 7.98 \\
\hline 76 & 189 & 113 & 1505.3 & 7.96 & 1503.2 & 7.95 \\
\hline 77 & 192 & 115 & 1524.8 & 7.94 & 1522.7 & 7.93 \\
\hline 78 & 195 & 117 & 1544.0 & 7.92 & 1542.0 & 7.91 \\
\hline 79 & 198 & 119 & 1563.2 & 7.89 & 1561.2 & 7.89 \\
\hline 80 & 201 & 121 & 1582.1 & 7.87 & 1580.3 & 7.86 \\
\hline
\end{tabular}




\begin{tabular}{|l|l|l|l|l|l|l|}
\hline 81 & 204 & 123 & 1600.9 & 7.85 & 1599.3 & 7.84 \\
\hline 82 & 207 & 125 & 1619.6 & 7.82 & 1618.1 & 7.82 \\
\hline 83 & 210 & 127 & 1638.1 & 7.80 & 1636.8 & 7.79 \\
\hline 84 & 213 & 129 & 1656.4 & 7.78 & 1655.4 & 7.77 \\
\hline 85 & 216 & 131 & 1674.6 & 7.75 & 1673.8 & 7.75 \\
\hline 86 & 219 & 133 & 1692.6 & 7.73 & 1692.1 & 7.73 \\
\hline 87 & 223 & 136 & 1716.6 & 7.70 & 1716.4 & 7.70 \\
\hline 88 & 226 & 138 & 1734.3 & 7.67 & 1734.4 & 7.67 \\
\hline 89 & 229 & 140 & 1751.8 & 7.65 & 1752.3 & 7.65 \\
\hline 90 & 232 & 142 & 1769.1 & 7.63 & 1770.1 & 7.63 \\
\hline 91 & 235 & 144 & 1786.3 & 7.60 & 1787.8 & 7.61 \\
\hline 92 & 238 & 146 & 1803.3 & 7.58 & 1805.4 & 7.59 \\
\hline 93 & 242 & 149 & 1826.1 & 7.55 & 1828.5 & 7.56 \\
\hline 94 & 245 & 151 & 1842.7 & 7.52 & 1845.8 & 7.53 \\
\hline 95 & 248 & 153 & 1859.2 & 7.50 & 1863.0 & 7.51 \\
\hline 96 & 251 & 155 & 1875.5 & 7.47 & 1880.1 & 7.49 \\
\hline 97 & 254 & 157 & 1891.7 & 7.45 & 1897.1 & 7.47 \\
\hline 98 & 258 & 160 & 1913.4 & 7.42 & 1919.4 & 7.44 \\
\hline 99 & 261 & 162 & 1929.2 & 7.39 & 1936.1 & 7.42 \\
\hline 100 & 264 & 164 & 1944.8 & 7.37 & 1952.7 & 7.40 \\
\hline
\end{tabular}

Figure 1: Binding energy per nucleon close to beta stability line of $Z=2$ to 100

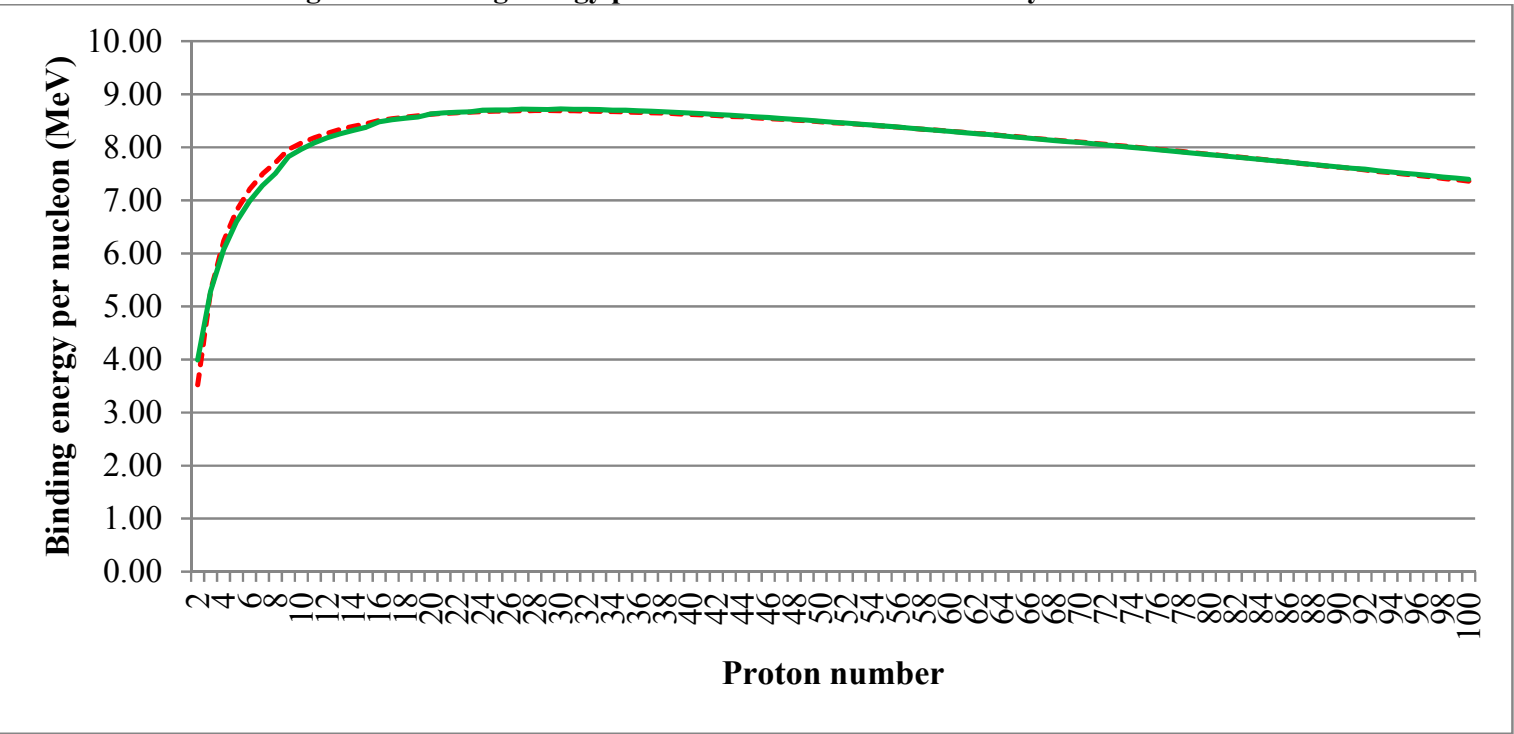

\section{References}

[1] U. V. S. Seshavatharam and S. Lakshminarayana. Understanding the basics of final unification with three gravitational constants associated with nuclear, electromagnetic and gravitational interactions. Journal of Nuclear Physics, Material Sciences, Radiation and Applications Vol-4, No-1, 1-19, (2017)

[2] Seshavatharam, U. V. S. \& Lakshminarayana, S., On the Basics of Nuclear Quantum Gravity. Prespacetime Journal. (2017). 8(6): 777-791.

[3] U. V. S. Seshavatharam et al. Understanding the constructional features of materialistic atoms in the light of strong nuclear gravitational coupling. Materials Today: 3/10PB, Proceedings 3 (2016) pp. 3976-3981

[4] U. V. S. Seshavatharam and S. Lakshminarayana. Final unification with three gravitational constants associated with nuclear, electromagnetic and gravitational interactions. International Journal of Advanced Astronomy. 4 (2) $105-109$ (2016) 
[5] U. V. S. Seshavatharam and S. Lakshminarayana. Scale independent workable model of final unification. Universal journal of physics and application. 10(6): 198-206, 2016

[6] U. V. S. Seshavatharam and S. Lakshminarayana. Towards a workable model of final unification. International Journal of Mathematics and Physics 7, No1,117-130 (2016).

[7] U. V. S. Seshavatharam and Lakshminarayana S. Final unification with Schwarzschild's Interaction. Journal of Applied Physical Science International 3(1): 12-22 (2015).

[8] Seshavatharam, U. V. S. and Lakshminarayana, S. The Possible Role of Newtonian, Strong \& Electromagnetic Gravitational Constants in Particle Physics. Prespacetime journal, Vol 7, Issue 5, pp. 857-888 (2016)

[9] U. V. S. Seshavatharam, Lakshminarayana S. Lakshminarayana. To Validate the Role of Electromagnetic and Strong Gravitational Constants via the Strong Elementary Charge. Universal Journal of Physics and Application 9(5): 210-219 (2015)

[10]U. V. S. Seshavatharam, Lakshminarayana S. To confirm the existence of nuclear gravitational constant, Open Science Journal of Modern Physics. 2(5): 89-102 (2015)

[11]U. V. S. Seshavatharam and S. Lakshminarayana. Understanding nuclear structure with Schwarzschild interaction and Avogadro number. Proceedings of the DAE-BRNS Symp. On Nucl. Phys. 60 (2015). F8, 852

[12]U.V. S. Seshavatharam and S. Lakshminarayana. Analytical estimation of the gravitational constant with atomic and nuclear physical constants. Proceedings of the DAE-BRNS Symp. On Nucl. Phys. 60 (2015) F7, 850

[13] C. Patrignani et al. (Particle Data Group), Chin. Phys. C, 40, 100001 (2016) and 2017 update

[14] Peter J. Mohr, David B. Newell, and Barry N. Taylor. CODATA recommended values of the fundamental physical constants: 2014. Rev. Mod. Phys. 88, 035009.

[15] G. Rosi, F. Sorrentino, L. Cacciapuoti, M. Prevedelli and G. M. Tino1. Precision measurement of the Newtonian gravitational constant using cold atoms. Nature 510, 518-521. (2014)

[16] S. Schlamminger and R.D. Newman. Recent measurements of the gravitational constant as a function of time. Phys. Rev. D 91, 121101 (2015)

[17] O. F. Akinto, Farida Tahir. Strong Gravity Approach to QCD and General Relativity. arXiv:1606.06963v3

[18] Roberto Onofrio. On Weak Interactions as Short- Distance Manifestations of Gravity. Modern Physics Letters A, Vol. 28, No. 7, 1350022 (2013)

[19] Roberto Onofrio. Proton radius puzzle and quantum gravity at the Fermi scale. EPL 104, 20002 (2013)

[20]Deaver, Bascom; Fairbank, William (July 1961). Experimental Evidence for Quantized Flux in Superconducting Cylinders. Physical Review Letters. 7(2): 43-46.

[21] The Periodic Table of the Elements (including Atomic Radius) www.sciencegeek.net/tables/AtomicRadius.pdf

[22] S. HAŁAS.100 years of work function. Materials Science, Poland, Vol. 24, No. 4, 2006

[23] Hector Javier Durand-Manterola. Dipolar Magnetic Moment of the Bodies of the Solar System and the Hot Jupiters. Planetary and Space Science 57:1405-1411 (2009)

[24] S. Hawking, Particle Creation by Black Hole, Commun. Math. Phys. 43, 199-220(1975)

[25] Helmut Satz. The Quark-Gluon Plasma.Nucl.Phys.A862-863:4-12,201.

[26] Sebastien Guillot et al. Measurement of the Radius of Neutron Stars with High S/N Quiescent Low-mass X-ray Binaries in Globular Clusters. Astrophys. J. 772 (2013)

[27] T. Bayram et al. New parameters for nuclear charge radius formulas. ACTA PHYSICA POLONICA B. Vol. 44,No 8, 1791-1799 (2013)

[28] I.Angeli, K.P. Marinovab. Table of experimental nuclear ground state charge radii: An update. Atomic Data and Nuclear Data Tables 99 (2013) 69-95.

[29] Angeli. A consistent set of nuclear rms charge radii: properties of the radius surface R(N,Z). Atomic Data and Nuclear Data Tables 87 (2004) 185-206

[30] Chowdhury, P.R. et al. Modified Bethe-Weizsacker mass formula with isotonic shift and new driplines. Mod. Phys. Lett. A20 p.1605-1618. (2005).

[31] N. Ghahramany et al. New scheme of nuclide and nuclear binding energy from quark-like model. Iranian Journal of Science \& Technology. 2011; A3: 201-208.

[32] N.Ghahramany et al. New approach to nuclear binding energy in integrated nuclear model. Journal of Theoretical and Applied Physics 6:3 (2012).

[33]U. V. S Seshavatharam, S. Lakshminarayana. Simplified Form of the Semi-empirical Mass Formula. Prespacetime Journal. (2017). 8(7): 881-810.

[34]U. V. S Seshavatharam, S. Lakshminarayana. On the role of strong coupling constant and nucleons in understanding nuclear stability and binding energy. Journal of Nuclear Sciences. (2017); 4(1): 7-18.

[35]U. V. S. Seshavatharam, Lakshminarayana S. Understanding Nuclear Stability, Binding Energy and Magic Numbers with Fermi Gas Model. Journal of Applied Physical Science International, 4 (2) pp.51-59 (2015). 
[36] U. V. S. Seshavatharam and S. Lakshminarayana. Understanding nuclear stability and binding energy with nuclear and electromagnetic gravitational constants. 61st DAE-BRNS Symposium on Nuclear Physics. A136. 332

[37] U. V. S. Seshavatharam, Lakshminarayana S. To unite nuclear and sub-nuclear strong interactions. International Journal of Physical Research, 5 (2) (2017) 104-108.

[38] W. D. Myers et al. Table of Nuclear Masses according to the 1994 Thomas-Fermi Model.(from nsdssd.lbl.gov)

[39] C. L. Morris et al. A new method for measuring the neutron lifetime using an in situ neutron detector. Report number: LA-UR-16-27352.https://arxiv:1610.04560.

[40]B. Andreas et al. An accurate determination of the Avogadro constant by counting the atoms in a $28 \mathrm{Si}$ crystal. Phys. Rev. Lett. 106, 030801.

[41] Massa, E.; Nicolaus, A. International determination of the Avogadro constant. Metrologia Volume: 48, Issue: 2, 2011.

[42]K. Becker, M. Becker and J. H. Schwarz. String Theory and M-theory: A Modern Introduction. Cambridge University Press, (2006)

[43] Edward Witten. What Every Physicist Should Know About String Theory. GR Centennial Celebration, Strings 2015, Bangalore, India. (2015).

[44] Brandenburg J. E. The GEM Unification Theory of the Vacuum: Did Dimensional Collapse Trigger the Big Bang. International Journal of Astrophysics and Space Science. Special Issue: Quantum Vacuum, Fundamental Arena of the Universe: Models, Applications and Perspectives. Vol. 2, No. 6-1, pp. 24-38. (2014). 